\title{
EIN KURZER ÜBERBLICK ÜBER DIE GESCHICHTE DER FLEDERMAUSFORSCHUNG
}

von

\author{
M. EISENTRAUT \\ Buschstr. 45, 53 Bonn, B.R. Deutschland
}

Es ist das hohe Verdienst unserer Kollegen aus der CSSR, den Anstoss zu einem Zusammentreffen und einer engeren Zusammenarbeit der Chiropteren-Forscher aus aller Welt gegeben zu haben. Sie ergriffen, wie Sie wissen, die Initiative und luden $1968 \mathrm{zu}$ der ersten internationalen Tagung in ihr Land ein. Es waren äussere Umstände, die es mit sich brachten, dass diese Tagung nur von wenigen Teilnehmern besucht werden konnte. Um so erfreulicher ist es, dass nun die zweite Tagung in Amsterdam eine so stattliche Zahl von Teilnehmern zusammengeführt hat. Sie sind dem Ruf unserer niederländischen Kollegen gefolgt, denen wir für die Einladung und für das Zustandekommen und die Organisation dieses Treffens besonderen Dank schulden.

Dass überhaupt ein solches Vorhaben geplant und verwirklicht werden konnte, ist zweifellos ein Zeichen dafür, dass in den letzten Jahrzehnten dieses Spezialgebiet der Mammalogie, die Chiropterologie, eine herausragende Bedeutung erlangt hat und sich eines besonderen Interesses bei den Säugetierkundlern erfreut.

Für mich selbst ist es eine Ehre, diese Konferenz mit einem Uberblick über die Geschichte der Fledertierforschung einzuleiten. Ich muss jedoch gestehen, dass ich nur zögernd an diese Aufgabe herangegangen bin, einen historischen Rückblick zu geben. Zum ersten ist es eine zeitraubende Sache, sich durch die Fülle der Literatur hindurchzuarbeiten. Zum anderen ist es in mancher Beziehung auch eine undankbare Aufgabe, denn wie soll ich jedem, der unser Wissen über die Fledertiere bereichert hat, gerecht werden? Ist die Gefahr nicht gross, dass ich, wenn ich Namen von Fledermausforschern nenne, den einen oder anderen vergesse oder nicht genügend hervorhebe? Und wenn es sich dann um einen Zeitgenossen handelt, so nimmt dieser mir begreiflicherweise mein Versäumnis übel.

Aus diesen Erwägungen heraus habe ich es daher vorgezogen, nur bei meinem Rückblick auf die ältere Vergangenheit wichtige Namen zu nennen, im übrigen aber bei der Abhandlung der jüngeren und jüngsten Zeit die Personen zugunsten der sachlichen Ausführungen weitgehend in den Hintergrund treten zu lassen. Es kann auch nicht erwartet werden, dass ich jede einzelne Arbeit würdige, es kommt mir vielmehr darauf an, in grossen Zügen den Verlauf der Entwicklung zu schildern und die einzelnen Epochen in der Geschichte der Fledermausforschung herauszuarbeiten. Wo soll ich beginnen?

Ich möchte die vor-linnésche Zeit kurz zusammenfassen als die Zeit der noch recht unklaren Vorstellungen über die Natur der Fledertiere, als eine Zeit, in der noch manche unsinnigen und abergläubischen Vorstellungen auch in ernst zu nehmenden Büchern herumspuken. Ich erwähne hier das bekannte Werk des Züricher Naturforschers Conrad Gesner, der von 1516-1565 gelebt hat und der wohl als erster die Naturgeschichte zur Wissenschaft erhoben hat. Es fehlt ihm aber noch der klare Artbegriff, und daher gelangt er nicht zu einer systematischen Anordnung, die ja die Voraussetzung für jede wissenschaftliche Aufbauarbeit ist. In seiner "Historia animalium" (1551/58) spricht er von einer Zwitterstellung der Fledermäuse zwischen Vogel und Säugetier. Er schreibt: "Die Fledermauss ist ein Mitteltier zwischen dem Vogel und der Mauss, also dass man sie billich eine fliegende Mauss nennen kann, wiewohl sie weder unter die Vögel noch unter die Mäuss kan gezehlet werden, dieweil sie beider Gestalt an sich hat". Er spricht dann von der "Gestalt dieses Vogels", von der "Speis dieses Vogels" 
und "was von diesem Vogel in der Arznei dem Menschen nützlich seye" und ergeht sich hier in der Aufzählung obskurer Rezepte für Arzneien aus Fledermäusen für die verschiedensten $\mathrm{Ge}-$ brechen.

Ein Zeitgenosse Gesners, der englische Zoologe Edward Wotton (1492-1555) erkannte dagegen schon klar und wohl als erster die wahre Natur der Fledermäuse und reiht sie unter die Quadrupeden, die Säugetiere, ein. Er darf als der bedeutendste Vorläufer Linnés angesehen werden.

Mit dem schwedischen Naturforscher Carl von Linné, der von 1707-1778 lebte, beginnt nun eine neue Zeit des naturwissenschaftlichen Denkens und systematischen Arbeitens. Er führte bekanntlich konsequent die binäre Nomenklatur durch und schuf ein für damalige Zeit übersichtliches System der Pflanzen und Tiere. Sein grundlegendes Werk "Systema naturae" erschien 1735 und erlebte in rascher Folge neue Auflagen. Es bedeutete für die beschreibende Naturwissenschaft den Beginn der wissenschaftlichen Systematik. Dies Werk kann daher auch für die Fledertierforschung als ein Anfang angesehen werden.

Linné stellte die Fledertiere anfangs zu den Raubtieren, später zu den Primaten. In der 1766 erschienenen 12. Auflage seines genannten Werkes teilte er die Primaten in vier Abteilungen ein: Homo, Simia, Lemur und Vespertilio. Unter letzteren führt er 6 Arten auf, für die er sämtlich den Gattungsnamen Vespertilio benutzt. Er kennt nur zwei europäische und vier aussereuropäische Vertreter. Zu ersteren gehören Vespertilio auritus, die heutige Plecotus auritus, und Vespertilio murinus, die auch heute noch diesen Namen trägt. Bei den vier aussereuropäischen Fledertieren handelt es sich um Vespertilio vampyrus von Asien, den heutigen Pteropus vampyrus, um Vespertilio spectrum von Amerika, den heutigen Vampyrus spectrum, ferner um Vespertilio perspicillatus von Amerika, den heutigen Artibeus perspicillatus oder jamaicensis, und schliesslich um Vespertilio spasma von Asien, die heutige Art Megaloderma spasma. Es ist bemerkenswert, dass Linné eine siebente Fledermausart kennt, nämlich Noctilio leporinus, das Hasenmaul oder die Fischerfledermaus von Amerika, die er jedoch als besondere Abteilung in die Gruppe der Nager einreiht, und zwar auf Grund der besonderen Stellung und Bildung der Schneidezähne, die auf den ersten Blick den Nagezähnen eines Rodentiers ähnlich sehen.

Linné und sein Werk sind ebenso wie für die anderen Tiergruppen auch für die Fledertiere Aus- gangspunkt einer nun folgende ausserordentlich fruchtbaren Zeit naturwissenschaftlichen Forschens.

Es beginnt die grosse Periode der Neuentdeckungen und Artbeschreibungen, die Zeit, in der ganz allgemein die systematische und faunistische Forschung einen gewaltigen Aufschwung nimmt, von dem auch die Chiropterologie ihren nicht geringen Anteil abbekommt. Es ist die Epoche der grossen Entdeckungsreisen, der Expeditionen in noch unbekannte und unerschlossene Gebiete unserer Erde. Es gelangte von diesen Reisen ein wertvolles wissenschaftliches Material in die $\mathrm{Mu}-$ seen, wo es von Spezialisten bearbeitet und determiniert wurde. Von den Männern, die während dieser etwa 100 Jahre währenden Zeit unsere Artenkenntnis der Chiropteren bereichert haben, sei es, dass sie selbst Reisen unternahmen, sei es, dass sie Reiseausbeuten bearbeiteten, nenne ich Peter Simon Pallas (1741-1811), Frédérik Cuvier (1773-1838), Etienne Geoffroy de Saint Hilaire (1772-1844), Thomas Horsfield (1773-1859), Coenraad Temminck (1778-1858), Eduard Eversmann (1794-1860), Moritz Wagner (1813 -1887), Karl Peters (1815-1867), Prinz Max von Wied (1782-1867), Eduard Rüppell (1794 -1884), George Waterhouse (1810-1888), John Edward Gray (1800-1875) und Theodor v. Heuglin (1824-1876).

Diese Bereicherung der Artenkenntnis, die nicht zuletzt auch die Ordnung der Chiropteren betrifft, zeigt sich in folgender Zusammenstellung:

Linné kannte, wie schon erwähnt, 7 Fledertierarten.

Schreber nennt in seinem 1775 erschienenen Werk "Die Säugetiere", 21 Arten, Koch in der Einleitung zu seiner Arbeit "Das Wesentliche der Chiropteren", 1863, bereits über 300 Arten und Dobson in seinem bekannten Chiropteren-Katalog, 1878, an die 400 Arten.

Für die Megachiropteren macht Andersen in seinem Katalog 1912 folgende Zahlenangaben: Es werden aufgeführt

- von Linné, 1758, in der 10. Auflage seiner Systema naturae 1 Art;

- von E. Geoffroy, 1810 (in : Description des Rousettes et des Céphalotes) 12 Arten;

- von Temminck, 1825, in seiner "Monographies de Mammalogia" 20 Arten und von dem gleichen Autor, 1837, 37 Arten;

- von Peters in seinem Buch über "Die Flederhunde", 1867, 49 Arten;

- von Gray in seinem Catalogue of the Monkeys, Lemurs and Fruiteating Bats, 1870, 57 Arten;

- von Dobson in seinem "Catalogue of the Chiroptera", 1878, 70 Arten;

- von Matschie in seinen "Chiroptera des Berliner Museums für Naturkunde", 1899, 120 Arten; und schlieBlich 
- von Andersen in seinem genannten MegachiropterenKatalog, 1912, 186 Arten mit 228 Formen.

Ich bin in dieser Zusammenstellung der Megachiropteren schon bis in unser Jahrhundert vorgedrungen und möchte betonen, dass die Neuentdeckungen und Neubeschreibungen von Arten, also die Vervollständigung unserer Formenkenntnis, eigentlich niemals zu einem Stillstand und Abschluss gekommen sind und bis in unsere Tage weiterlaufen. Auch heute noch sind an den grossen Museen der Welt Chiropteren-Spezialisten tätig und betreiben ihre systematischen Untersuchungen. Ich möchte aber betonen, dass in der Vergangenheit sicherlich zu viel Formen beschrieben wurden; manche Namen sind inzwischen auch bereits in die Liste der Synonyma aufgenommen.

Wenn man die von Walker, 1964, in seinem Werk "Mammals of the world" genannten Arten zusammenzählt, so kommt man auf annähernd 800 , von denen knapp ein Fünftel Megachiropteren betrifft und die übrigen der Unterordnung der Microchiropteren angehören.

Ich habe hier zunächst die weltweite systematische Fledertier-Forschung kurz behandelt. Es liegt aber vielleicht nahe, auch einen Blick auf die faunistische Erforschung der europäischen Fledermäuse zu werfen, die viele von uns ganz besonders interessiert. Diese hat nun ganz zweifellos eine hohe Blütezeit in der ersten Hälfte des vorigen Jahrhunderts gehabt. Wenn wir die Artnamen der europäischen Fledermäuse durchsehen, so finden wir eine ganze Anzahl, bei deren Benennung verdiente Chiropteren-Forscher Pate gestanden haben:

Myotis daubentoni, nattereri, bechsteini; Nyctalus leisleri; Pipistrellus nathusii, savii; Miniopterus schreibersi; Eptesicus nilssoni; Rhinolophus blasii, mehelyi.

Von den Forschern, die sich durch Neuentdeckung und -Beschreibung von europäischen Arten oder durch gröszere Zusammenfassungen verdient gemacht haben, nenne ich:

Schreber, mit 6 neuen Arten; Kuhl, mit 7 Arten; Blasius \& Kayserling, mit 3 Arten; Bechstein, mit 1 Art; Bonaparte, mit 2 Arten.

In die Reihe bekannter damaliger Fledermausforscher gehören auch Friedrich, Anton Kolenati und Carl Koch. Beim Lesen ihrer zusammenfassenden Arbeiten, die teilweise in etwas entlegenen Zeitschriften erschienen sind, fühlt man die Begeisterung und Liebe, mit denen sie über Fledermäuse gearbeitet haben, und es setzt uns immer wieder in Erstaunen, mit welcher Genauig- keit und Sorgfalt diese Männer gearbeitet und sich bemüht haben, feine Unterschiede auch innerhalb der einzelnen Formengruppen herauszufinden.

Mit den genannten Forschern schliesst zunächst die grosse Zeit der faunistischen Untersuchungen über europäische Fledermäuse ab. Wir können mögen mir verzeihen, wenn ich hier die europäschen oder auch euerasiatischen Chiropteren-Forschung bezeichnen. Unsere Freunde aus Ubersee mögen mir verzeihen, wenn ich hier die europäischen Belange etwas in den Vordergrund gestellt habe. Hinsichtlich der weltweiten Erforschung der Fledertiere möchte ich daher aus der Fülle der Namen wenigstens noch einige aus der jüngeren Zeit herausgreifen: Oldfield Thomas, Gerrit Miller, G. H. H. Tate und Glover Allen.

Nach dieser Blütezeit systematischer Forschung trat nun - sieht man von der schon erwähnten laufend fortschreitenden Erweiterung unserer Artenkenntnis $a b$ - eine gewisse Ruhezeit ein. Es waren andere Disziplinen der Zoologie, die in den Vordergrund rückten. Im Zuge der technischen Errungenschaften - ich denke dabei zum Beispiel an die Fortentwicklung des Mikroskops gewannen an Bedeutung die Histologie, die Cytologie, insbesondere die Vererbungscytologie, die sich dann mit der experimentellen Vererbungsforschung vereinigt, ferner die Physiologie mit ihren Unterdisziplinen, wobei zunächst die Chiropteren kaum als Untersuchungsobjekte herangezogen wurden. Dies bedeutet allerdings nicht, dass die Wissenschaft die Fledermäuse ganz vergessen hätte, jedoch ist die Zahl der Arbeiten, die sich mit Chiropteren beschäftigen, in der Folgezeit nicht allzu gross. Ich erwähne die anatomischen Arbeiten von Macalister (1872) über die Muskulatur und von Robin (1881) allgemein über die Anatomie der Fledertiere, von Schöbl (1871) über die Flughaut und von Levy (1912) über die Flugmuskulatur. Ferner nenne ich die Arbeiten von Duval (1895) über die Embryologie, von Rollinat und Trouessart (1896) uiber die Fortpflanzung der Fledermäuse, nachdem schon vorher von $\mathrm{Pa}$ genstecher (1859), von Van Beneden (1875) und anderen die bemerkenswerte Erscheinung der Ovulationsverzögerung bei unseren heimischen Fledermäusen bekannt geworden war.

Erst mit Beginn der dreissiger Jahre unseres Jahrhunderts erfährt nun die Fledertierforschung neue Impulse, und es kommt zu einer starken Wiederbelebung des Interesses an dieser Säugetierordnung, gewissermassen zu einer Wiederentdeckung der Fledertiere für die Wissenschaft. Das 
Interesse richtet sich dabei zunächst in erster Linie auf biologisch-ökologische Fragen, und es ist bemerkenswert, dass bisweilen von ganz verschiedenen Seiten her die gleichen Probleme in Angriff genommen wurden, ein Zeichen dafür, dass wieder einmal die Zeit für solche Untersuchungen reif war und es nur noch eines Anstosses bedurfte. Denn bereits in den zwanziger Jahren nimmt die Zahl vor allem biologischer Arbeiten und Beobachtungen an Fledermäusen zu, und zwar oft im Rahmen allgemeiner säugetierkundlicher Forschungen. So ist z.B. in Russland mit Beginn der sowjetischen Epoche der Name Sergej Iwanowitsch Ognew mit einer aufblühenden Säugetierforschung aufs engste verbunden.

Anfang der dreissiger Jahre wurden annähernd gleichzeitig in den USA und in Europa Markierungsversuche an Fledermäusen im grossen Umfang in Gang gesetzt. Ich darf vielleicht hier einmal kurz schildern, wie ich selbst in diese Arbeitsrichtung hineingekommen bin, nur als Beispiel dafür, wie weit solche Unternehmungen auch von Zufälligkeiten und glücklichen Umständen abhängig sind. Ich arbeitete damals in Berlin über den Winterschlaf der Säugetiere und hatte Hamster, Haselmaus, Siebenschläfer und Igel untersucht und dabei gesehen, dass es recht grosse Unterschiede im Ablauf dieser saisonbedingten Lethargie-Erscheinung bei den einzelnen Arten gibt. Nun wollte ich auch den Winterschlaf der Fledermäuse kennenlernen. Aber woher Fledermäuse bekommen? Da traf es sich gut, dass im Berliner Museum der Säugetierspezialist Dr. Schwarz arbeitete, der durch befreundete Beziehungen Zugang zu der Zitadelle in Spandau hatte. Bei dem gemeinsamen ersten Besuch im Dezember 1931 lernte ich dann zum ersten Mal winterschlafende Fledermäuse kennen; es waren Mausohren, Wasser- und Fransenfledermäuse. Das Interesse war geweckt, und ich überlegte, wie ich zu weiteren Fledermausarten kommen könne. Von meinen sommerlichen Exkursionen kannte ich in der Nähe von Berlin ein Kalkbergwerk und wusste, dass hier unterirdische Stollen vorhanden waren. Ich konnte erwarten, dass diese Stollen während des Winters möglicherweise Fledermäusen als Winterquartier dienten. Als ich dann im Winter 1931/32 die erste Exkursion in dieses Gebiet unternahm, war ich völlig überrascht und geradezu fasziniert von der Fülle von Fledermäusen, die hier überwinterten. Ich schätzte den Bestand an Myotis myotis auf etwa 5000; dazu kamen einige Hundert Myotis dauhentoni, ferner in geringerer Zahl Myotis mysta- cinus, nattereri, bechsteini, dasycneme, Plecotus auritus, Barbastella barbastellus und Pipistrellus pipistrellus. Hier eröffneten sich mir also ungeahnte Beobachtungs- und Untersuchungsmöglichkeiten, von denen eine dann auch die Markierung der Fledermäuse zwecks Untersuchung ihrer Wanderungen und all der anderen Fragen, die damit verbunden sind, betraf.

Fast gleichzeitig wurde in Amerika durch Griffin und andere mit planmässigen Beringungen begonnen, nachdem dort schon in früheren Jahren Einzelversuche in dieser Richtung unternommen worden waren. Ich möchte meinen, dass diese Markierungsversuche, die inzwischen in vielen Ländern Eingang gefunden haben, nicht unwesentlich dazu beigetragen haben, dass sich junge Zoologen für die Fledermausforschung begeisterten und nun sich diesem Arbeitsgebiet mit allem Eifer zuwandten.

Wie erwähnt, waren es vor allem biologischökologische Arbeiten, die nun in grosser Zahl und in vielen Ländern erschienen und noch bis zum heutigen Tage erscheinen. Es sind Arbeiten über den Winterschlaf und die Besonderheiten des Wärmehaushaltes, über die Fortpflanzung und Brutpflege der Fledermäuse und über die Ortstreue, die saisonbedingten Wanderungen, das Heimfindevermögen und über die Lebensdauer, eine Frage, die einzwischen zu erstaunlichen und kaum erwarteten Ergebnissen geführt hat und die nur mit Hilfe der Markierungsmethode angegangen werden kann.

Gleichzeitig tauchte aber erneut auch die Frage des Fledermausschutzes auf, die schon ältere Fledermausforscher bewegte und die gerade heute nicht ernst genug genommen werden kann. In diesem Zusammenhang möchte ich noch einmal auf die Beringungsarbeiten zurückkommen und sehr eindringlich vor einer nicht zu verantwortenden Ubertreibung der Beringungsunternehmungen warnen. Die Markierung darf nicht zum Selbstzweck werden, sondern ist nur Mittel zum Zweck. Ich fühle mich deshalb zu dieser Warnung veranlasst, weil durch nichtsachgemässe Ausführung der Beringung oder durch zu häufige oder zeitlich ungünstige Kontrollen der Fledermausquartiere Schaden angerichtet werden kann. Es geht mir fast so wie dem Zauberlehrling in Goethes Gedicht, der sich vor den Wassermassen, die der Besen herbeischleppt, nicht mehr retten kann, weil er das erlösende Zauberwort vergessen hat und nun klagt: "Die ich rief, die Geister, werd ich nun nicht los". 
Ganz besonders in den Vordergrund des allgemeinen Interesses wurden die Fledermäuse dann durch die Entdeckung der Echopeilung (Echolocation) gerückt. Auch diese Forschungsrichtung begann in den 30 ger Jahren, und zwar wiederum von zwei unabhängig von einander arbeitenden Seiten. Einmal durch den Holländer Dijkgraaf, zum anderen durch den Amerikaner Griffin und seine Mitarbeiter. Es sind dies Untersuchungen, die bis zum heutigen Tage weitergeführt wurden, insbesondere von Griffin und Möhres und deren Schülern. Es ist jedoch nicht meine Aufgabe, hier im einzelnen auf die schönen Untersuchungen und überraschenden Ergebnisse einzugehen, die Ihnen allen ja ohnehin bekannt sind. Ich möchte nur darauf hinweisen, dass diese Arbeiten anknüpfen an die alten hochinteressanten aber lange Zeit wieder in Vergessenheit geratenen Versuche des italienischen Physiologen Lanzarro Spalanzani am Ende des 18. Jahrhunderts. Ich habe hier Spalanzani genannt, weil ich meine, dass, wenn überhaupt Namen genannt werden, sein Name bei einem Uberblick über die Geschichte der Fledermausforschung nicht vergessen werden sollte.

In dieser mit den dreissiger Jahren beginnenden ausserordentlich fruchtbaren Periode der Chiropterenforschung sind inzwischen eine Reihe zusammenfassender Werke entstanden, und zwar in einer ganzen Anzahl verschiedener Länder und Sprachen. Bitte haben Sie Verständnis, wenn ich es mir versage, einzelne Namen zu nennen, denn wir wollten ja in erster Linie die Forschungsperioden herausarbeiten und die zeitgenössischen Ein- zelpersonen in den Hintergrund treten lassen.

Ich komme damit zum Ende und möchte zum Abschluss einen kurzen Ausblick geben. Wir wissen nicht, ob diese letzte Periode der Fledertierforschung ihren Höhepunkt überschritten hat, ob nun die Chiropteren wieder ein wenig in den Hintergrund treten werden. Vielleicht tauchen neue Fragen auf, die der Forschung wieder einen Antrieb geben. Wichtig wird es sein, auf dem systematischen Sektor zu einer Zusammenfassung zu kommen und in die Fülle der beschriebenen Formen und in die verwandtschaftlichen Beziehungen nach den Methoden moderner Systematik Klarheit zu bringen, wie dies bereits hier und da begonnen wurde. Ich denke dabei an die Gattung Pteropus oder an Pipistrellus oder an die Hipposideros cafter-Gruppe um nur einige Beispiele zu nennen. Weiterhin ist es notwendig, unsere Kenntnis von der Lebensweise der Arten zu vervollständigen, denn von vielen kennen wir auch heute noch kaum mehr als Namen und Aussehen. Manche allgemeinen Fragen sind noch offen, wie beispielsweise das Sozialverhalten der Fledertiere. Ein noch nicht gelöstes Problem ist das der Fernorientierung der Fledermäuse, das ich selbst noch gern in Angriff genommen hätte, wozu mir aber bisher Zeit und Gelegenheit gefehlt haben.

Eines darf ich wohl abschliessend noch einmal sagen: Diese letzte Forschungsperiode hat die Chiropteren als die zweitgrösste Säugetierordnung in ein besonderes Blickfeld gerückt. Dies beweist ja auch die hohe Zahl von Zoologen, die sich mit Chiropteren befassen. 